\section{Case Report}

Korean J Transplant 2021;35:116-123 https://doi.org/10.4285/kjt.20.0037

\section{$\mathrm{KJT}^{<}$ OF TRANSPLANTATION}

\title{
Salvage living donor liver transplantation for post-resection recurrence of combined hepatocellular carcinoma-cholangiocarcinoma
}

\author{
Minjae Kim, Shin Hwang, Gi-Won Song, Chul-Soo Ahn, Deok-Bog Moon, \\ Dong-Hwan Jung, Gil-Chun Park, Sung-Gyu Lee
}

Division of Hepatobiliary Surgery and Liver Transplantation, Department of Surgery, Asan Medical Center, University of Ulsan College of Medicine, Seoul, Korea

Received September 2, 2020

Revised November 19, 2020

Accepted November 20, 2020

Corresponding author: Shin Hwang Department of Surgery, Asan Medical Center, University of Ulsan College of Medicine, 88 Olympic-ro 43-gil, Songpagu, Seoul 05505, Korea

Tel: +82-2-3010-3930

Fax: +82-2-3010-6701

E-mail: shwang@amc.seoul.kr

(c) The Korean Society for Transplantation This is an Open Access article distributed under the terms of the Creative Commons Attribution Non-Commercial License (http://creativecommons.org/licenses/ by-nc/4.0/) which permits unrestricted non-commercial use, distribution, and reproduction in any medium, provided the original work is properly cited.
Salvage liver transplantation (LT) is a definite treatment for recurrent hepatocellular carcinoma (HCC) after hepatectomy. Combined hepatocellular carcinoma-cholangiocarcinoma (cHCC-CC) is not eligibly indicated for LT because of high post-transplant recurrence. We present a case of salvage living donor liver transplantation (LDLT) in a patient with tumor recurrence after surgical resection of CHCC-CC. A 61-year-old male patient diagnosed with chronic hepatitis $\mathrm{B}$ underwent right posterior sectionectomy for $\mathrm{HCC}$. The pathological diagnosis revealed presence of a $3.2-\mathrm{cm}$-sized $\mathrm{CHCC}-\mathrm{CC}$ with stem cell features and intermediate cell-subtype. Intrahepatic tumor recurrence occurred 9 months later and transarterial chemoembolization was performed. Due to the progress of recurrent tumors, ABO-incompatible LDLT was performed. The explant liver pathology revealed four small masses of $\mathrm{CHCC}-\mathrm{CC}$ with stem cell features. Pulmonary metastasectomy and chest wall resection were performed for metastatic lesions at 10 months after LT. Multiple tumor recurrence was detected at 21 months after LT with progression despite sorafenib administration. Currently, the patient is alive for past 26 months after LT. In conclusion, the clinical sequences of this case suggest that the role of salvage $\mathrm{LT}$ for $\mathrm{CHCC}-\mathrm{CC}$ is much more limited than that for HCC because the tumor biology of CHCC-CC is more aggressive compared with HCC.

Keywords: Hepatectomy; Tumor marker; Neoadjuvant therapy; Recurrence; Tumor biology

\section{INTRODUCTION}

Salvage liver transplantation (LT) is defined as LT for hepatocellular carcinoma (HCC) recurrence following prior hepatectomy for HCC or post-hepatectomy liver failure. Primary liver tumors other than HCC, such as intrahepatic cholangiocarcinoma (ICC) and combined hepatocellular carcinoma-cholangiocarcinoma (cHCC-CC) are usually not indicated for LT because of the high risk of posttransplant tumor recurrence [1]. Although we possess considerable experience of LT with ICC and CHCC, most of the tumors had been misdiagnosed as HCC before transplantation. In principle, pathologically confirmed ICC or CHCC-CC has not been regarded as an eligible indication of LT in our institution. On the contrary, very early ICC or CHCC-CC, which is one or two tumors measuring less than $2 \mathrm{~cm}$ in size, can be considered as a potential indication of LT because the posttransplant risk of tumor recurrence has been reported to be relatively low $[1,2]$.

In Korea, salvage living donor liver transplantation 


\section{HIGHLIGHTS}

-We present a case of salvage living donor liver transplantation in a patient with tumor recurrence after surgical resection of combined hepatocellular carcinoma-cholangiocarcinoma (cHCC-CC).

- The clinical sequences suggest that the role of salvage liver transplantation for CHCC-CC is much more limited than that for hepatocellular carcinoma because of more aggressive tumor biology.

(LDLT) for recurrent HCC after hepatic resection has been performed more frequently than before. However, so far, salvage LT for postresection recurrence of $\mathrm{CHCC}-\mathrm{CC}$ has not been reported in the literature. Considering that the number of patients with pathologically confirmed $\mathrm{cH}$ $\mathrm{CC}-\mathrm{CC}$ has been gradually increasing, transplant surgeons may face patient's demand for asking salvage LDLT to treat recurrent $\mathrm{CHCC}-\mathrm{CC}$. Thus, we herein present our experience on salvage LDLT performed in a patient who had tumor recurrence after surgical resection of $\mathrm{CHCC}-\mathrm{CC}$.

\section{CASE REPORT}

This study was approved by the Institutional Review Board of Asan Medical Center (IRB No. 2020-0836), and the requirement for informed consent was waived.

A 61-year-old male patient diagnosed with chronic hepatitis B was admitted to our institution considering the presence of liver mass detected during routine HCC surveillance. A 2-cm-sized single mass was located in the liver segment VII, which was diagnosed as HCC or ICC on dynamic computed tomography (CT) and single-contrast liver magnetic resonance imaging (MRI) (Fig. 1). There was an ill-defined mild hypermetabolic lesion in the right posterior section of the liver on fluorodeoxyglucose-positron emission tomography (FDG-PET). The level of serum alpha-fetoprotein (AFP) was $4.3 \mathrm{ng} / \mathrm{mL}$; proteins induced by vitamin $\mathrm{K}$ antagonist or absence-II (PIVKA-II) was $20 \mathrm{mAU} / \mathrm{mL}$, and carbohydrate antigen 19-9 (CA 19-9) was $27 \mathrm{ng} / \mathrm{mL}$. Percutaneous liver biopsy revealed HCC. Subsequently, the patient underwent right posterior sectionectomy. The final pathologic diagnosis was a $3.2-\mathrm{cm}$-sized cHCC-CC with stem cell features and intermediate-cell subtype. Microvascular invasion was present (Fig. 2A). This lesion was tumor stage II (T2NOMO) according to the 8th edition of the American Joint Committee on Cancer (AJCC)
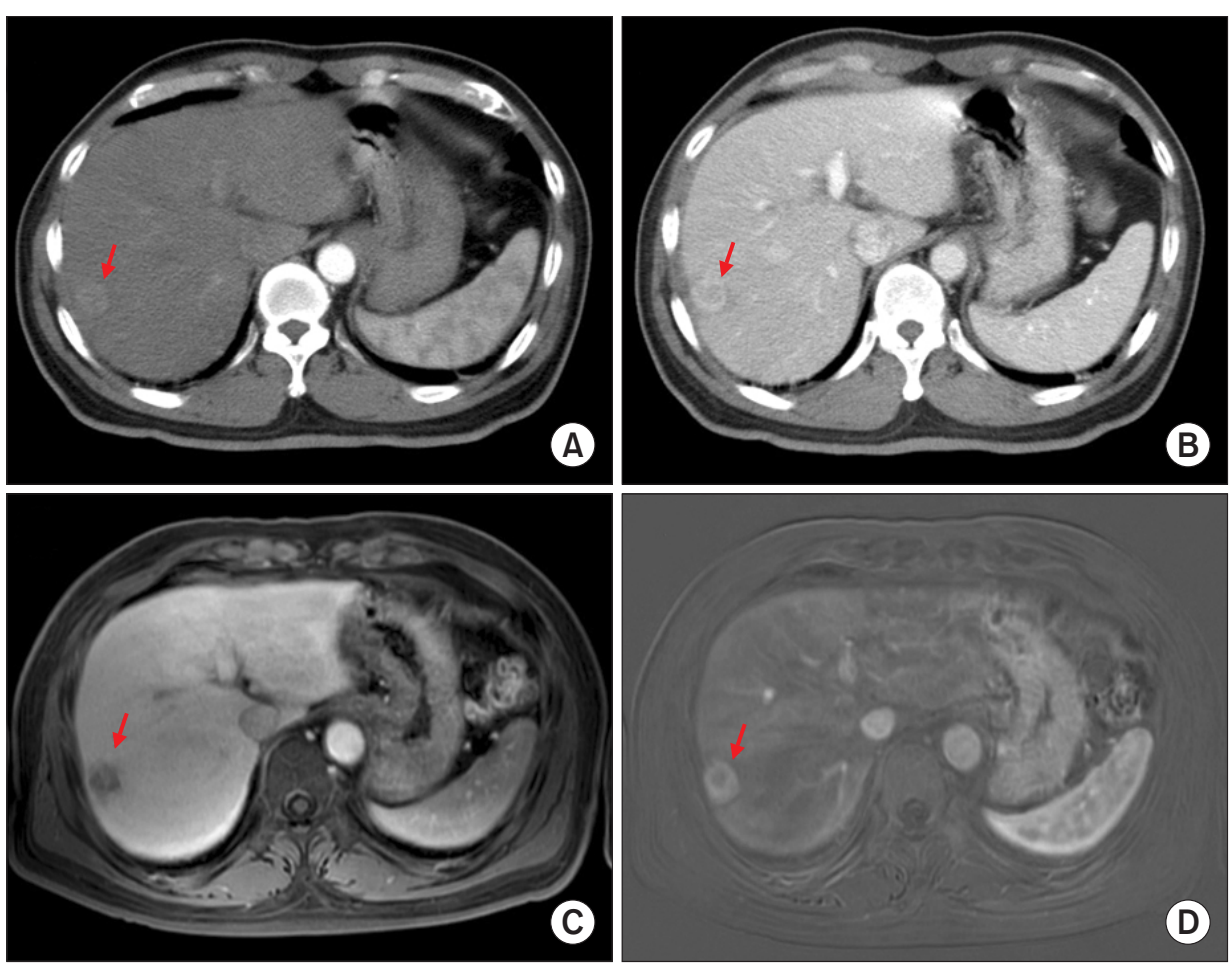

Fig. 1. Initial preoperative imaging studies. Dynamic computed tomography $(A, B)$ and magnetic resonance imaging $(C, D)$ show a 2-cm-sized single mass (arrows) located in the liver segment VII with a diagnosis of hepatocellular carcinoma or intrahepatic cholangiocarcinoma. 

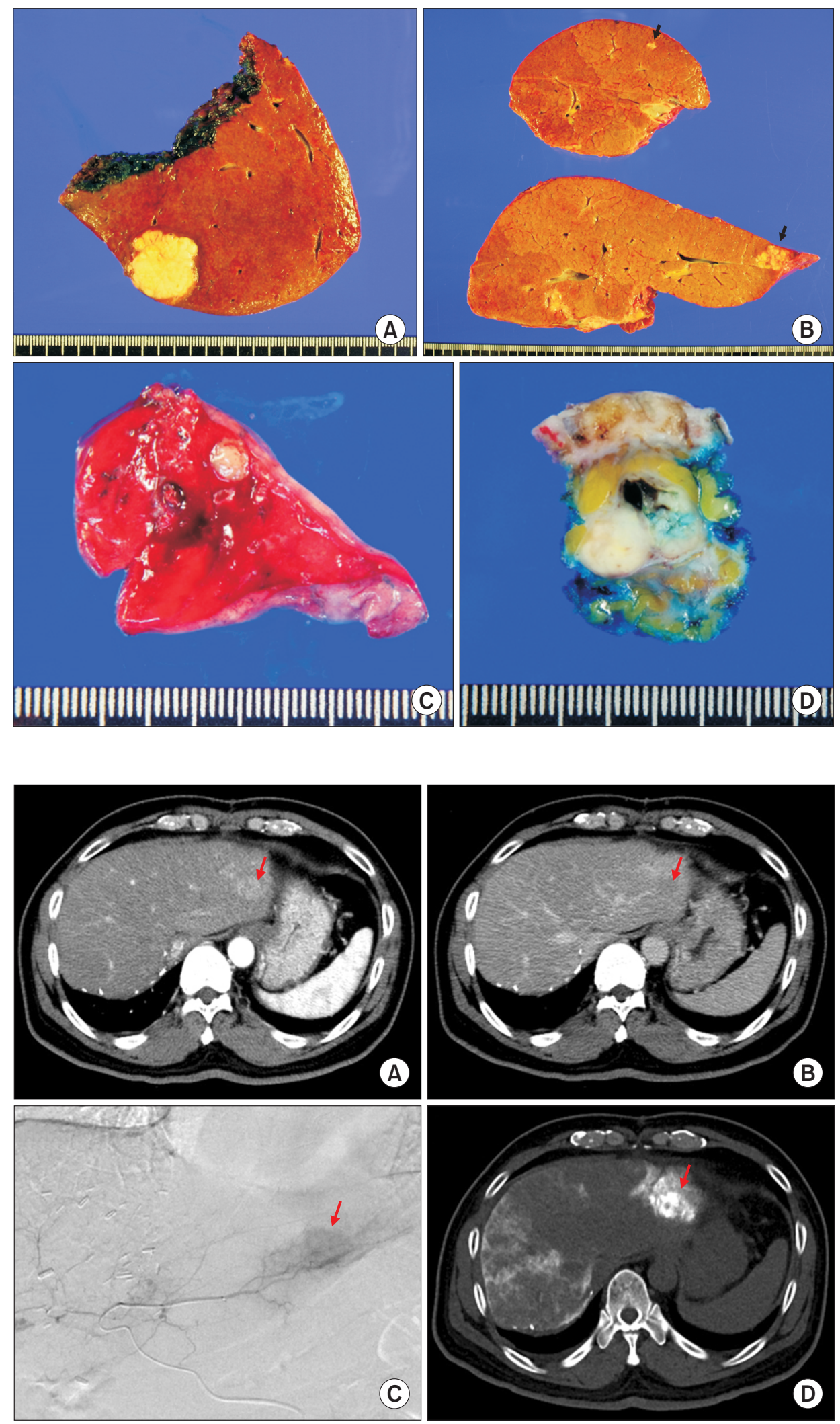

Fig. 2. Gross photograph of the resected specimens. (A) Liver specimen following right posterior sectionectomy shows a 3.2-cm-sized tumor. (B) The explant liver specimen shows the presence of multiple tumors (arrows). The resected specimens from the lung (C) and chest wall (D) show metastatic tumors.

Fig. 3. Imaging study findings of tumor recurrence taken at 9 months after hepatic resection. Computed tomography images show a recurrent intrahepatic mass in the liver segment II (A, B). (C) Transarterial chemoembolization shows hypervascular tumor staining. (D) Computed tomography scan taken 2 days later shows diffuse lipiodol uptake at the mass. Arrows indicate the recurrent mass. 

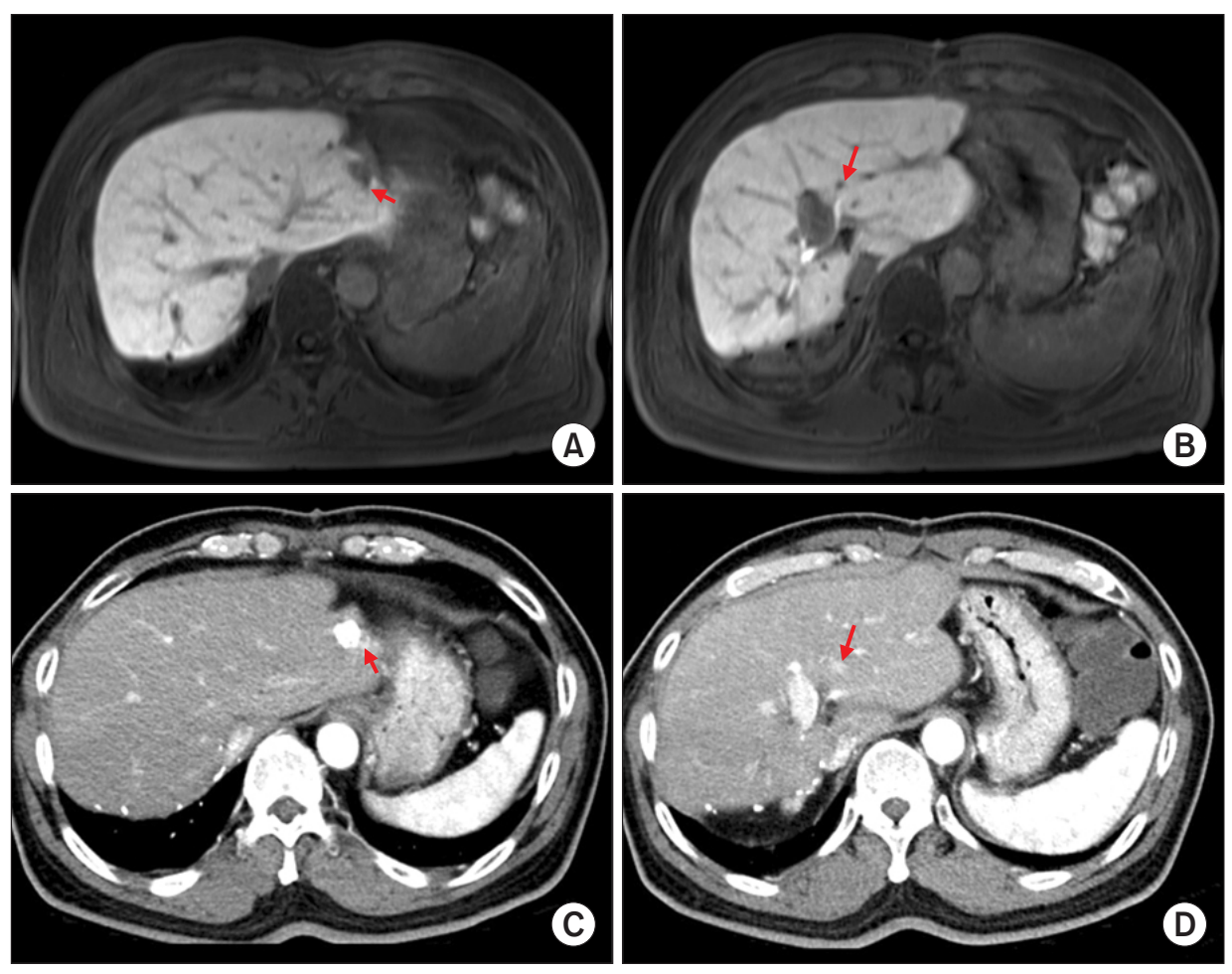

Fig. 4. Pretransplant imaging study findings of the recurrent tumors. Liver magnetic resonance imaging taken 10 months after hepatic resection shows two masses at the liver segments II and III (A, B). There was no noticeable progression of the two recurrent lesions on liver computed tomography taken 1 month later (C, D). Arrows indicate the recurrent masses.
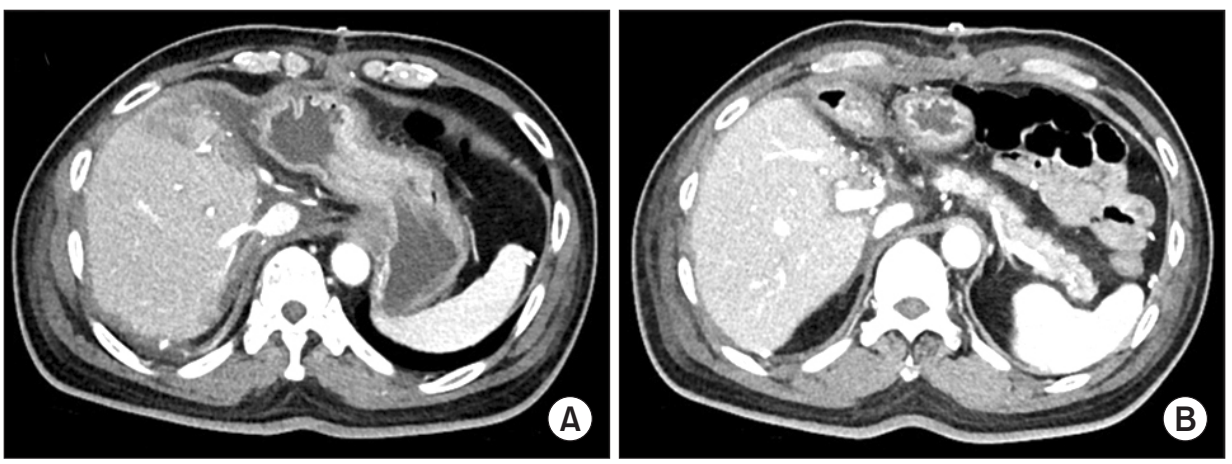

Fig. 5. Early posttransplant imaging study findings. Computed tomography images taken 3 weeks after transplantation show no abnormal findings at the arterial $(A)$ and portal (B) phase images.

system. The patient recovered uneventfully from hepatic resection.

Recurrent intrahepatic mass was detected in the liver segment II in the follow-up liver CT taken 9 months after hepatic resection. Accordingly, transarterial chemoembolization (TACE) was performed, in which tumor staining was visible. CT scan taken 2 days later showed diffuse lipiodol uptake at the mass (Fig. 3).

Liver MRI taken at 11 months after hepatic resection revealed the presence of two masses in the liver segments II and III. There was no noticeable progression of the two recurrent lesions on liver CT (Fig. 4). At that time, the level of AFP was $16.8 \mathrm{ng} / \mathrm{mL}$; PIVKA-II was $38 \mathrm{mAU} / \mathrm{mL}$, and CA
19-9 was $35.3 \mathrm{ng} / \mathrm{mL}$. The tumor responded partially to TACE and occurrence of a new lesion was observed. Consequently, we decided to perform salvage LDLT.

The donor was the 34-year-old son of the patient. As ABO blood groups were not compatible, the patient was treated with rituximab and exchange plasmapheresis. ABO-incompatible LDLT using a modified right liver graft of $653 \mathrm{~g}$ was performed at 11 months after prior hepatic resection. The graft-recipient weight ratio was $0.94 \%$. The patient recovered uneventfully from the LDLT operation (Fig. 5).

The final pathologic diagnosis of the explant liver was the presence of four masses of CHCC-CC with stem cell 
features and intermediate-cell subtype, and the size of the tumor was $2.8 \mathrm{~cm}, 0.9 \mathrm{~cm}, 0.5 \mathrm{~cm}$, and $0.3 \mathrm{~cm}$, respectively. Microvascular invasion was present without perineural invasion (Fig. 2B). These lesions were tumor stage II (T2NOMO) according to the 8th edition of the AJCC system.

On CT scans taken 8 months after LDLT, a nodule was identified in the right lower chest wall, which was diagnosed with metastatic $\mathrm{CHCC}-\mathrm{CC}$ on percutaneous needle biopsy. Another small nodule was also identified in the upper lobe of the right lung on FDG-PET and chest CT
(Fig. 6A-D). After diagnosis of tumor recurrence, the primary immunosuppressant was changed from tacrolimus monotherapy to a combination therapy with everolimus, mycophenolate mofetil and minimal tacrolimus. Everolimus monotherapy was theoretically desirable, but its immunosuppressive potency and efficacy were not high enough, especially during the first year after LT. Everolimus and mycophenolate mofetil were used as the main immunosuppressants, whereas minimal tacrolimus was used to match with the social health insurance policy.

At 10 months after LDLT, pulmonary metastasectomy
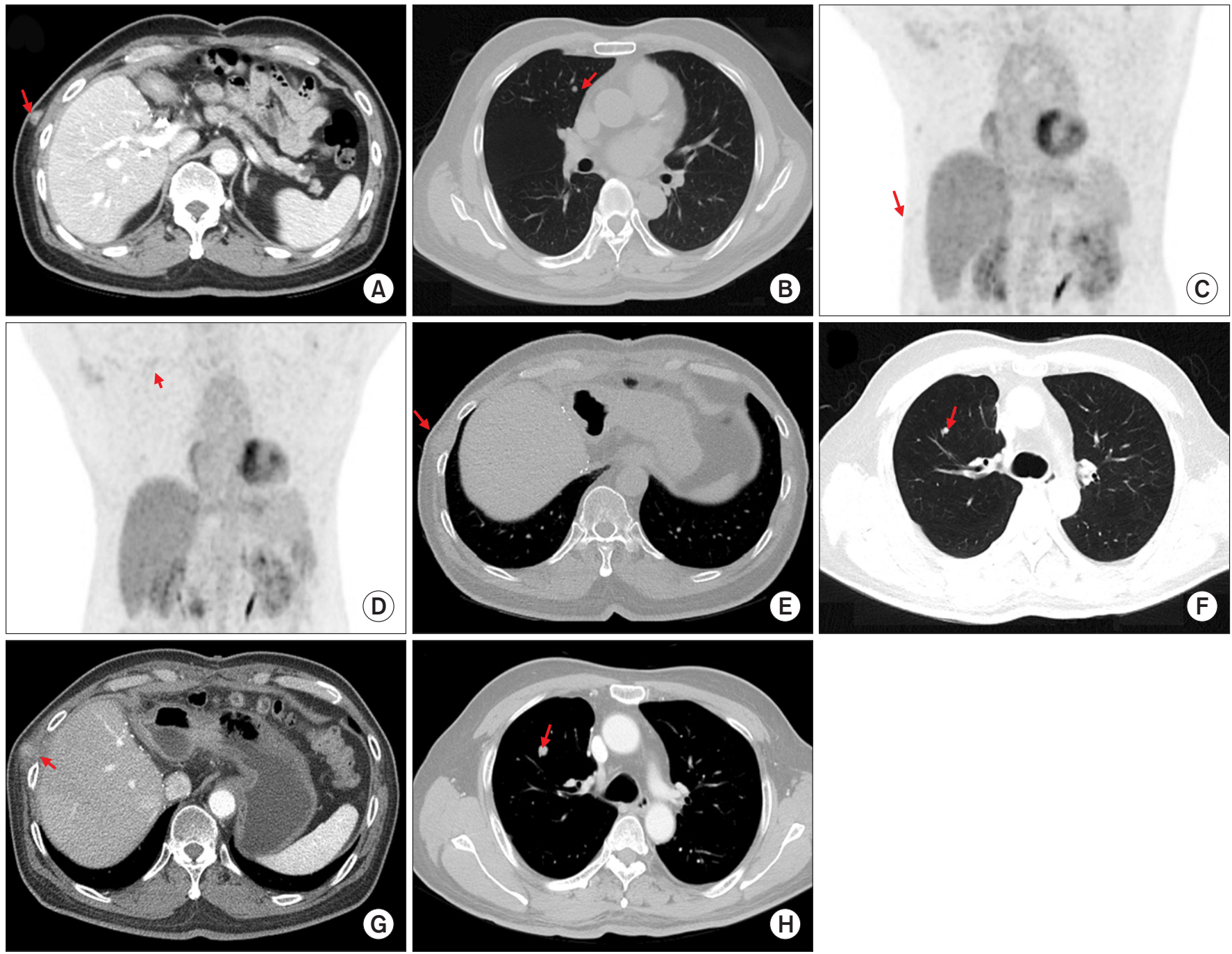

Fig. 6. Post-transplant imaging study findings of the recurrent tumors. Computed tomography taken 8 months after transplantation shows a nodule in the right lower chest wall (A). A small nodule is identified in the upper lobe of the right lung in the chest computed tomography (B). These two recurrent lesions are visible in the fluorodeoxyglucose-positron emission tomography $(C, D)$. Arrows indicate the recurrent masses. Progression of the recurrent lung and chest wall tumors is observed in the images taken at 21 months $(E, F)$ and 24 months $(G, H)$ after transplantation, respectively. Arrows indicate the recurrent masses. 
and chest wall resection were concurrently performed. The lung and subcutaneous lesions were identified as metastatic tumors (Fig. 2C and D). At 21 months after LDLT, multiple recurrent lesions were identified in the right lung and right lower chest wall, and the CT scan taken at 24 months revealed slow progression of these recurrent tumors (Fig. 6E-H). Sorafenib was administered to the patient. This patient is currently alive for the past 26 months with normal liver function and ordinary day-to-day performance.

\section{DISCUSSION}

The $\mathrm{CHCC}-\mathrm{CC}$ is an uncommon type of primary liver cancer containing components of both HCC and ICC. In our previous study on surgical resection of primary liver tumors, this disease is a rare tumor, comprising approximately an incidence of $5.8 \%$ [3]. In the literature, CHCC-CC accounts for $0.8 \%$ to $14.3 \%$ of primary liver malignancies, with incidences widely varying among studies [4]. According to a population-level analysis in the United States, 52,825 patients had HCC, 7,181 patients had ICC, and 465 patients had CHCC-CC between 1988 and 2009; thus, the proportion of cHCC-CC was $0.8 \%$ [5].

After the evolution of classification of CHCC-CC twice in 1949 and 1985, the current classification, 2010 World Health Organization (WHO) classification includes two main histological forms as the classical type and subtypes with stem cell features [6]. The classical type meets the traditional definition of CHCC-CC. Subtypes with stem cell features are further classified as typical, intermediate-cell, and cholangiolocellular subtypes. We previously presented the postresection prognosis in 100 cases of cHCC-CC [3], in which the presence of stem cell features was closely associated with favorable tumor biology. In the recently updated 2019 WHO classification of tumors of the digestive system $[7,8]$, the classical type of $\mathrm{CHCC}-\mathrm{CC}$ persists, but the subtype of cHCC-CC with stem cell features is no longer used. There are two subtypes that reflect the concurrent pathological components such as CHCC-CC-intermediate cell carcinoma with immunohistochemical findings showing a mixture of both hepatocytic and cholangiocytic features on a cell-by-cell basis, and cHCC-CC-cholangiolocellular carcinoma with HCC component. Thus, the pathology of our patient was re-classified as CHCC-CC-intermediate cell carcinoma according to the 2019 WHO classification.
The recurrence rate of $\mathrm{cHCC}-\mathrm{CC}$ after hepatic resection is high, thus various treatments have been used for recurrent lesions, including liver-directed therapy and systemic chemotherapy. TACE has been frequently performed for recurrent cHCC-CC lesions, but its therapeutic effect is debatable because of the histological heterogeneity of cHCC-CCs and more fibrotic and less vascular nature compared with HCC [9]. The efficacy of TACE on primary unresectable and recurrent $\mathrm{CHCC}-\mathrm{CC}$ s was investigated in two studies from our institution, concluding that the treatment response and prognosis are highly related to tumor vascularity $[10,11]$. The role of systemic chemotherapy for unresectable and recurrent $\mathrm{CHCC}$-CCs remains unclear and has been identified to be associated with unfavorable outcomes. A multicenter study involving 36 patients evaluated several first-line treatments including gemcitabine/ cisplatin, fluorouracil/cisplatin, and sorafenib. Treatment with sorafenib monotherapy showed poorer overall survival compared to treatment with platinum-containing regimens [12]. In our patient, TACE was performed for the recurrent tumor, but only partial response was observed and a majority of the tumor portion remained viable.

LT has been performed in the case of a small number of patients with CHCC-CC. We previously reported $32 \mathrm{LT}$ cases with $\mathrm{CHCC}-\mathrm{CC}$; a majority of them were diagnosed with HCC before LT and concurrent HCC was identified in the explant liver in 12 patients [3]. Posttransplant tumor recurrence and patient survival rates were $15.6 \%$ and $84.4 \%$ at 1 year and $32.2 \%$ and $65.8 \%$ at 5 years, respectively. Patients with very early stage CHCC-CC ( 1 or 2 tumors $\leq 2.0$ $\mathrm{cm}$ ) showed $13.3 \%$ tumor recurrence and $93.3 \%$ patient survival rates at 5 years. Based on this experience, we have not considered pathologically confirmed cHCC-CC as an eligible indication of LT because very early stage cHCC-CC is usually misdiagnosed as HCC before hepatic resection or LT, thus not being diagnosed as CHCC-CC.

Introduction of mammalian target of rapamycin inhibitor (mTOR) inhibitors has made noticeable changes in immunosuppressive regimens. Everolimus has been covered by social health insurance since early 2016 in Korea. So far, we do not consider everolimus to be one of the primary immunosuppressants, thus it has not been frequently used in our institution yet. Everolimus can be administer after first 1 month of LT primarily because of risk of hepatic artery thrombosis and wound healing problems. Korean Social Health Insurance policy forcefully suggests administrating tacrolimus and everolimus concurrently [13]. The main indication of everolimus in our institution includes 
development of HCC recurrence or de novo malignancy because $\mathrm{mTOR}$ inhibitors are known to be the only immunosuppressive agent with anti-tumor effect [14]. Because of social health insurance policy and its relatively low immunosuppressive potency, it is usually administered concurrently with tacrolimus or mycophenolate. Everolimus monotherapy has been adopted, especially in only patients with overt presentation of primary tumor recurrence or de novo malignancy.

The postresection and post-transplant prognoses of CHCC-CC are dependent on the AJCC system. The 8th edition of the AJCC tumor system for ICC is applied to cHCC-CC because this staging system is equally valid for ICC, CHCC-CC, and primary endocrine tumor of the liver [15]. In our patient, the primary tumor identified during prior hepatic resection was a T2 lesion (solitary tumor with vascular invasion) and the recurrent tumor was also a T2 lesion (multiple tumors with or without vascular invasion). Although two small recurrent lesions were identified at the pretransplant imaging workup, there were four masses in the explant liver. This patient experienced multiple extrahepatic metastases at 10 months after LDLT. Although metastasectomy and systemic chemotherapy were performed, the progression of the recurrent tumors was still observed. Considering these aggressive posttransplant sequences, we presume that the clinical course after LT might not be better compared with non-LT treatment for post-resection recurrence of CHCC-CC. We would like to emphasize that salvage LDLT for recurrent CHCC-CC after hepatic resection should not be attempted because the tumor biology of CHCC-CC is much more aggressive than that of ordinary HCC.

In conclusion, the clinical sequences of this case and our previous experience of LT suggest that the role of salvage $\mathrm{LT}$ for $\mathrm{CHCC}-\mathrm{CC}$ is much more limited than that for $\mathrm{HCC}$ because the tumor biology of CHCC-CC is more aggressive compared with HCC.

\section{ACKNOWLEDGMENTS}

\section{Conflict of Interest}

Shin Hwang was an editorial board member of the journal but did not involve in the peer reviewer selection, evaluation, or decision process of this article. No other potential conflicts of interest relevant to this article were reported.

\section{Funding/Support}

This study was supported by research grant from the Korean Society for Transplantation (2020-00-03005-009).

\section{ORCID}

Minjae Kim

Shin Hwang

Gi-Won Song

Chul-Soo Ahn

https://orcid.org/0000-0001-6743-0636 https://orcid.org/0000-0002-9045-2531

Deok-Bog Moon https://orcid.org/0000-0002-4235-0434

Dong-Hwan Jung

Gil-Chun Park https://orcid.org/0000-0002-3844-3646 https://orcid.org/0000-0002-8209-3540 https://orcid.org/0000-0001-5984-023X

Sung-Gyu Lee https://orcid.org/0000-0003-1631-3258 https://orcid.org/0000-0001-9161-3491

\section{Author Contributions}

Conceptualization: SH. Data curation: GWS, CSA, DBM, DHJ, GCP. Formal analysis: SH. Methodology: DHJ, GCP. Project administration: SGL. Visualization: SH. Writingoriginal draft: $\mathrm{SH}, \mathrm{MK}$. Writing-review \& editing: $\mathrm{SH}$.

\section{REFERENCES}

1. Jung DH, Hwang S, Song GW, Ahn CS, Moon DB, Kim $\mathrm{KH}$, et al. Longterm prognosis of combined hepatocellular carcinoma-cholangiocarcinoma following liver transplantation and resection. Liver Transpl 2017;23:330-41.

2. Sapisochin G, Facciuto $M$, Rubbia-Brandt L, Marti J, Mehta N, Yao FY, et al. Liver transplantation for "very early" intrahepatic cholangiocarcinoma: international retrospective study supporting a prospective assessment. Hepatology 2016;64:1178-88.

3. Jung DH, Hwang S, Hong SM, Chung YK, Song GW, Lee $\mathrm{YJ}$, et al. Post-resection prognosis of combined hepatocellular carcinoma-cholangiocarcinoma according to the 2010 WHO classification. World J Surg 2017;41:1347-57.

4. Lee JH, Chung GE, Yu SJ, Hwang SY, Kim JS, Kim HY, et al. Long-term prognosis of combined hepatocellular and cholangiocarcinoma after curative resection comparison with hepatocellular carcinoma and cholangiocarcinoma. J Clin Gastroenterol 2011;45:69-75.

5. Garancini M, Goffredo P, Pagni F, Romano F, Roman S, Sosa JA, et al. Combined hepatocellular-cholangiocarcinoma: a population-level analysis of an uncommon primary liver tumor. Liver Transpl 2014;20:952-9. 
6. Theise ND, Park YN, Nakanuma Y. Combined hepatocellular-cholangiocarcinoma. In: Bosman FT, Carneiro F, Hruban RH, Theise ND, eds. WHO classification of tumours of the digestive system. 4th ed. Lyon, Fr: IARC; 2010. p. 225-7.

7. Brunt E, Aishima S, Clavien PA, Fowler K, Goodman Z, Gores $\mathrm{G}$, et al. CHCC-CCA: consensus terminology for primary liver carcinomas with both hepatocytic and cholangiocytic differentation. Hepatology 2018;68:11326.

8. Sempoux C, Kakar S, Kondo F, Schirmacher P. Combined hepatocellular-cholangiocarcinoma. In: Bosman FT, Carneiro F, Hruban RH, Theise ND, eds. WHO classification of tumours: digestive system tumours. 5 th ed. Lyon, Fr: IARC; 2019. p. 260-2.

9. De Vito C, Sarker D, Ross P, Heaton N, Quaglia A. Histological heterogeneity in primary and metastatic classic combined hepatocellular-cholangiocarcinoma: a case series. Virchows Arch 2017;471:619-29.

10. Kim JH, Yoon HK, Ko GY, Gwon DI, Jang CS, Song HY, et al. Nonresectable combined hepatocellular carcinoma and cholangiocarcinoma: analysis of the response and prognostic factors after transcatheter arterial chemo- embolization. Radiology 2010;255:270-7.

11. Na SK, Choi GH, Lee HC, Shin YM, An J, Lee D, et al. The effectiveness of transarterial chemoembolization in recurrent hepatocellular-cholangiocarcinoma after resection. PLoS One 2018;13:e0198138.

12. Kobayashi S, Terashima T, Shiba S, Yoshida Y, Yamada I, Iwadou S, et al. Multicenter retrospective analysis of systemic chemotherapy for unresectable combined hepatocellular and cholangiocarcinoma. Cancer Sci 2018;109:2549-57.

13. Kang SH, Hwang S, Ha TY, Song GW, Jung DH, Ahn CS, et al. Cross-sectional analysis of immunosuppressive regimens focused on everolimus after liver transplantation in a Korean high-volume transplantation center. Korean J Transplant 2019;33:98-105.

14. Yamanaka K, Petrulionis M, Lin S, Gao C, Galli U, Richter $\mathrm{S}$, et al. Therapeutic potential and adverse events of everolimus for treatment of hepatocellular carcinoma: systematic review and meta-analysis. Cancer Med 2013;2:862-71.

15. Amin MB, Edge S, Greene F, Byrd DR, Brookland RK, Washington $\mathrm{MK}$, et al. AJCC cancer staging manual. 8th ed. New York, NY: Springer; 2017. 